\title{
(2) OPEN ACCESS \\ Recommendations by the European Network of Paediatric Research at the European Medicines Agency (Enpr-EMA) Working Group on preparedness of clinical trials about paediatric medicines process
}

\author{
Angeliki Siapkara, ${ }^{1}$ Claudio Fracasso, ${ }^{2}$ Gunter F Egger, ${ }^{3}$ Carmelo Rizzari, ${ }^{4}$ \\ Cristina Serén Trasorras, ${ }^{5}$ Dimitrios Athanasiou, ${ }^{6}$ Mark A Turner 이, ${ }^{7}$ Working Group \\ Membership
}

${ }^{1}$ VRNN, Medicines and Healthcare products Regulatory Agency (MHRA), London, UK ${ }^{2}$ Pediatric Centre of Excellence, Pfizer S.r.l, Rome, Italy

${ }^{3}$ Scientific Evidence Generation Department, European

Medicines Agency, Amsterdam, Netherlands

${ }^{4}$ Department of Paediatrics, University of Milan-Bicocca, Milano, Italy

${ }^{5}$ Instituto de Investigación Sanitaria de Santiago de Compostela, Santiago de Compostela, Spain

${ }^{6}$ Word Duchenne Organization, Amsterdam, Netherlands ${ }^{7}$ Institute if Life Course and Medical Sciences, Liverpool Health Partners, University of Liverpool, Liverpool, UK

Correspondence to Prof Mark A Turner, Institute of Life Course and Medical Sciences, Liverpool Health Partners, University of Liverpool, Liverpool L69 3BX, UK; mark.turner@liverpool.ac.uk

Received 15 December 2020 Revised 19 March 2021 Accepted 20 March 2021

\section{ABSTRACT}

Conduct of clinical trials in babies, children and young people is often hindered by issues that could have been foreseen before the trial opened; that is, some clinical trials are often underprepared. In order to identify a good approach to trial preparedness, the European Network of Paediatric Research at the European Medicines Agency formed a working group. The Working Group included representation from regulators, industry, academics, paediatric clinical research networks and parents. The Working Group consulted widely about how to prepare for paediatric clinical trials. The Group's detailed recommendations have been published (https://www. ema.europa.eu/en/documents/other/preparednessmedicines-clinical-trials-paediatrics-recommendationsenpr-ema-working-group-trial_en.pdf).

This paper is a summary of the key recommendations including the following: start early, preferably in parallel to designing the medicine's development plan and individual protocols; identify the rationale and clinical need; listen to the perspectives of children and families, and of patient advocacy groups; identify how many people will be eligible for the trial; identify the resources needed, such as clinical facilities (including play therapy) and out-of-pocket expenditure by participants and their families; use all available data to estimate what is possible; present information about preparedness in a structured way; deploy proportionate resources to support the preparation of trials.

A well-prepared, well-designed trial is likely to require fewer changes during its course, be run in a shorter time frame and achieve expected objectives.

\section{INTRODUCTION}

Children deserve to be treated with high-quality medicines based on robust scientific information. ${ }^{12}$ Despite many improvements, including the introduction of new regulations, ${ }^{3-6}$ the availability of medicines for children is suboptimal because of the lack of relevant clinical trials due to the difficulties in implementing and conducting these trials. ${ }^{78}$ The number of eligible paediatric patients is often limited, and this requires particular attention to trial design. ${ }^{1910}$ Patients and their parents may be reluctant to enrol into a trial for many reasons. ${ }^{11-14}$ Research sites often overestimate what is possible

\section{What is already known on this topic?}

- Clinical trials are often poorly prepared.

- Poor preparation can lead to avoidable delays.

What this study adds?

- A structured approach to trial preparation integrates information from multiple sources.

- This report provides practical suggestions based on learning from industry, regulators, sites and patient advocates.

both in terms of recruitment and efforts needed to follow trials with an enhanced level of attention for the safety of a vulnerable population. Sites and other groups often underestimate the efforts required to run a trial (resources and time) and the burden of a trial experienced by study participants and their families. Drug companies, regulators and ethics committees can have different views about what should be done during drug development. ${ }^{15-17}$ Insufficient consideration of these complexities at the planning stage of a trial leads to delays in the delivery of trial results or sometimes even failure of the trial with potential loss of new therapy opportunities for the paediatric population. Experience suggests that some of these difficulties can be addressed before a trial opens. ${ }^{18} 19$ Discussion in 2016 between stakeholders under the remit of the European Network of Paediatric Research at the European Medicines Agency (Enpr-EMA) ${ }^{20}$ suggested that a shared framework for preparing trials is needed. Accordingly, a document ${ }^{21}$ was developed by a Working Group of Enpr-EMA ${ }^{22}$ which sets out recommendations for discussions about trial preparedness in paediatrics.

We define trial preparedness as a structured assessment of the key factors that could increase the likelihood of a smooth and timely course of a paediatric clinical trial, integrating information from multiple stakeholders on what is possible within individual studies and therefore also for the overall drug development plan within which a trial is embedded. Trial 'feasibility' is the likelihood of 
completing a trial in a timely manner. This document moves beyond the definition of feasibility to present a global determination of all aspects of a trial that need to be prepared.

One significant factor of preparedness is the study design, but this is not the only one. By design, we mean the selection of methods to answer a research question. When working with the paediatric population, it is essential to establish explicitly the rationale of the benefit of the research question for children. In parallel, trial design needs to take account of the specificities of neonates, infants, children and young people while maximising the use of extant data (including preclinical data such as toxicity) and minimising the burden of research in these populations. ${ }^{23} 24$ Many additional factors may play a role in designing a trial, such as the target disease, the available data and the phase of development, and most of them cannot be easily standardised within a guideline document. Thus, trial design is not discussed further in the document.

The recommendations in the Enpr-EMA document target companies and other organisations responsible for organising trials (sponsors) as well as people whose work includes the preparation of trials or the review trials before they open. The recommendations will be relevant to investigators and people with a range of functions including clinical trial operations, clinical staff who work on trials (doctors, nurses and pharmacists) and administrators. In addition, the document will be relevant to people who review clinical trials in companies, academic and clinical institutions, patient advocacy groups, regulators, research ethics committees and research infrastructures. However, it should be noted that the document does not describe all aspects of 'sponsor readiness', such as operational aspects within sponsors and intermediary organisations, for example, contract research organisations (CRO), or strategic factors, such as patient need and economic opportunities. Furthermore, activities relevant to development of age-appropriate formulations to pharmaceutical quality standards, as well as activities to support marketing of products, are important factors influencing paediatric trials but are out of the scope of the document.

\section{PROCESS TO DEVELOP THE GUIDELINE}

In order to support the development of these recommendations, the group collated together all existing resources, such as current regulatory guidance, outputs from previous initiatives and Enpr-EMA working groups, and published literature. In addition, the team sought to collect the experience and suggestions from different stakeholders by developing a survey and performing direct interviews.

A wide range of stakeholders, including sponsors, investigators, patient organisations, regulators and paediatric clinical research networks, was included to provide the broadest spectrum of knowledge and experience. The stakeholders answered an extensive questionnaire covering questions about four different areas of the planning and conduction of a paediatric clinical trial: planning phase, preparation of the study, study conduct and the poststudy aspects. Finally, an adapted version of the survey was shared with young people's advisory groups.

The key messages identified during these surveys and interviews have been included in the main body of the document and supported the conclusions of the proposed document. A detailed publication of these research findings is under preparation.

\section{Recommendations and principles of good preparation}

For the majority of paediatric clinical trials, problems can be addressed by using all available data to estimate what is possible using a structured approach. Adequate preparation, however, cannot remove all of the difficulties or estimate achieved patient numbers with complete accuracy. However, a well-prepared, well-designed trial is likely to require fewer changes during its course, be run in a shorter time frame and achieve expected objectives with the forecasted costs.

Trial preparation should be initiated before, and conducted in parallel to, the designing of the development plan and the individual trials, and also to sponsor readiness. Then trial conduct is often improved iteratively, by learning during the execution of a trial.

Planning any trial starts from considering whether there is sufficient scientific rationale and a real clinical need to answer a specific research question. It remains critical to define clearly a meaningful trial objective and whether this addresses a relevant paediatric unmet need. To this aim, the perspective of children and families, and of patient advocacy groups, can make significant contributions about the design and the organisation of the trial. This perspective is critical in developing a successful trial as it can have a direct impact on recruitment and the feasibility of a study.

Very often, limited resources are allocated in this early stage to support trial preparation because of the uncertainties on the effective execution of the trial, but remuneration for well-conducted preparedness activities may represent a worthwhile investment to facilitate the high-quality conduct of trials (including recruitment figures and complete data sets), thereby avoiding expenditure for poorly conducted, inconclusive trials and development plans.

The principles of good preparation are described in the full guidance document (https://www.ema.europa.eu/en/documents/ other/preparedness-medicines-clinical-trials-paediatrics-recommendations-enpr-ema-working-group-trial_en.pdf) and are summarised in box 1 .

\section{Structured justification of preparation}

The description of preparedness needs to be based on explicit data sources and explicit reasoning and can be modified iteratively. See box 2 for a possible structure for a justification of preparation.

As new information becomes available, the trial plan should be updated and the implications on the development plan reassessed. This could allow evidence and data-driven discussions between sponsors and regulators and will contribute to the development of realistic expectations and reduce the risk of infeasible trials. Any assumptions on the number of children available for recruitment should be made explicit and justified.

The construction of a flow diagram in terms of patient availability is critical to define the real target population, from epidemiology to eligibility (figure 1) and from eligibility to contents of locked database. It is important to ensure all assumptions made during the construction of a flow diagram are explicit.

Trials that may be used for regulatory purposes, that is, a paediatric label or for authorising a paediatric indication, need to enrol a patient population that will demonstrate an effect of the medicine while determining an acceptable safety profile. For these reasons, clinical trials in rare diseases need to draw on a global patient population to overcome small patient pools in any given country.

\section{Site contributions to preparedness}

Sites and networks of sites should be involved as early as possible in those aspects of trial preparation that they can contribute 
Box 1 Summary principles of good preparation (for full

list please refer to full guidance document)

\section{Principles}

- Develop a time-sensitive understanding of the context for planning of the trial (how many sites (with facilities required by the trial), how many participants at each site and costs of the trial).

- Contributions to preparedness can be data, estimates, judgements or opinions, but all should be verifiable.

- Look for sources of data and state methods used to find information (be aware of potentially different uses of the same terms for conditions and diseases in different contexts) including

i. Literature data on disease prevalence (including reviews, case reports and disease registries as applicable).

ii. Preclinical evidence.

iii. Population-based registry.

iv. Patient registry.

v. Drug registry.

vi. Real-life data repositories, electronic health records.

vii. Site data.

viii. Paediatric research networks or initiatives.

- Use opinion from experts based on experience, including nurses, study coordinators and physicians from all sites, not just large teaching hospitals. Supplement this with a small number of opinion leaders.

- Take into account the available data on the natural history (including prognosis) of the condition and relevant subsets of the condition under investigation when assessing the number, location and readiness of potential participants.

- Develop awareness of other trials that may lead to competition for resources or recruits, or opportunities for coenrolment.

- Take account of clinical reality across all trial sites. Variation in clinical practices across countries and between therapeutic areas should be considered.

- Identify the factors that are critical to the quality of the trial and the risks that threaten the integrity of these critical factors.

- Identify ethical and legal issues of the research and responses to potential questions/objections (eg, direct benefit, risk minimisation, child assent and confidentiality), taking account of differences across regions.

- Ensure appropriate development and availability of ageappropriate formulations based on target populations.

- Account for the social-economic status of the research locations, particularly for international trials.

- Focus on the burden on participants and their families.

i. Attendance at trial visits for the child, such as time, inconvenience, impact on school and leisure activities (when possible, use of available technology by participants at home).

ii. Parent/caregivers' burdens of a child's participation in a trial, including effects on work and the possibility to reimburse costs.

iii. Clinical burden on patient on top of standard treatment (eg, blood sampling)

- Carefully plan the time course of the trial. Do not assume a

linear rate of recruitment, particularly at site opening.
Box 1 Continued

- Consider need to gather data that supports health technology assessment and reimbursement decision, integrated with, or in parallel to, clinical development.

- Involve sites and networks (including clinical and methodological expert groups), patients and patient advocacy groups to promote the quality of protocol and process design (including information leaflet and consent forms).

- Seek regulatory input as early as possible (eg, on trial design, the need for age-appropriate formulations, on preclinical trials or other regulatory requirements).

- Aim for global alignment of the contributions to preparedness, but equally the contributions should reflect the diversity needed in elements of trial preparedness.

- Conduct clinical trial simulations: in silico and in clinical simulation facilities. Update preparedness work in case of significant delay or interfering event that may have affected the relevance of the previous simulation.

- Justify why the sample size required by the trial design is compatible with the number of participants that can realistically be expected to be recruited to the trial. In any case, other innovative methods should be explored to facilitate the generation of data in the most efficient way.

- Expend adequate effort on preparation that is proportionate to its benefits.

- Establish good communication between all parties involved, including investigators, patient organisations and experts in the disease as well as regulators early during the planning of the trial.

to. This work is separate from clinical work, and the sponsor should derive high value from the work of the sites, taking into consideration potential conflicts of interest. Early consultation aimed to prevent unfeasible procedures or work flows that are incompatible with standard care is critical as well as ongoing dialogues to ensure any changes to the study are accommodated by all relevant vendors. Sites play a key role in identifying local specificities, which directly impact on the design of the trial (ie, standard of care and vaccination schedule). Roles and responsibilities need to be clearly defined, and the organisation of the sites has to meet industry and regulatory standards.

It is extremely important that sites contribute to preparation for individual clinical trials by working on organisational aspects that are common to all clinical trials, such as monitoring organisation, facilities, personnel availability, and clear definition of roles and responsibilities. This generic work would facilitate site assessment of specific clinical trials.

\section{Participant contributions to preparedness}

The perspectives of potential participants are central to the preparation of trials. Children and young people have specific needs and views that have complex dynamics during acute and chronic illness. Early consultation with patients' and children's advocacy groups, ideally consultation with patient/parent/caregivers panels and community advisory boards, will improve the communication with the target population and allow the identification of potential practical barriers for the conduct of the trial. Their input should be heeded as far as possible and should include but not only limited to relevant endpoints, time of assessments, quality or life effects, tolerance of tests and assessments, impact on their daily life and family dynamics. 
Box 2 Exemplar of a structured outline of preparedness assessment

1. Statement of starting point: therapeutic need, clinical indication, development and availability of suitable ageappropriate dosage form(s), aim of plan/trial including regulatory purpose and scope of information needs.

2. Availability of participants.

a. Patient flow diagram annotated with sources of information and estimates of variation, particularly at key decision points.

b. Sensitivity analysis of patient availability.

3. Sites.
a. Availability of suitable sites with relevant expertise in clinical research.
b. Extent of modifications needed to sites.
c. Estimates of participants at each site that can be validated.
d. Account for other competing trials.
e. Availability of human resources at site level.

4. Completeness of data.

a. Retention of participants, based on acceptability of key trial assessments including what the expected retention is anticipated to be.

b. Sensitivity analysis of data completeness.

5. Implications.

a. Trade-off between need for information and availability of participants.

b. Areas of concern, anticipated weak links in the preparation.

c. Uncertainties in assumptions being made.

d. Actions required to optimise setup and conduct.

e. Actions required to maximise recruitment and retention.

Protocols should be made flexible enough to reflect this input as much as possible. Of course, these decisions have to be put in the frame of scientific and regulatory acceptability; therefore, it may be beneficial to obtain also regulatory input on the impact that these changes may have on the objectives and results of the research. Presenting planned trials at meetings of patients' associations, holding webinars and/or planning for periodic newsletters dedicated to patients during and after the trial, and lay summaries of trial results should be considered good practices for a full involvement of the families participating in the clinical trials, enhancing their role as participant and not only passive recipient of an experimental therapy. Support from patients' associations before, during and after the trial can also be helpful for trial participants and can increase participant retention in compliance with local regulations. Feedback to children, young people and families who contribute to trial preparation is essential, and sponsors need to plan how and when to provide this.

Advocacy groups can contribute to the preparation of plans and trials with

- Training of people who supply their contributions.

- Considering relevant endpoints, including, where possible, biomarkers and validated scales, time points of assessment and quality of life effects.

- Communication with patient community and awareness on new drug development (including age-appropriate dosage form when applicable).

- Review and contribution to creation of some trial-related documents (eg, consent/assent, information/awareness documents and lay summaries).

\section{Implications for sponsors}

Trial sponsors need to think ahead to include work on preparedness in all processes. CROs and other external vendors (such as data coordination centres, central laboratories, biobanks and drug suppliers) should be included in the assessment of preparedness. Sponsors should anticipate, allocate, deploy and expend relevant resources to meet the needs of good preparedness. Industry should design adult programmes and trials to
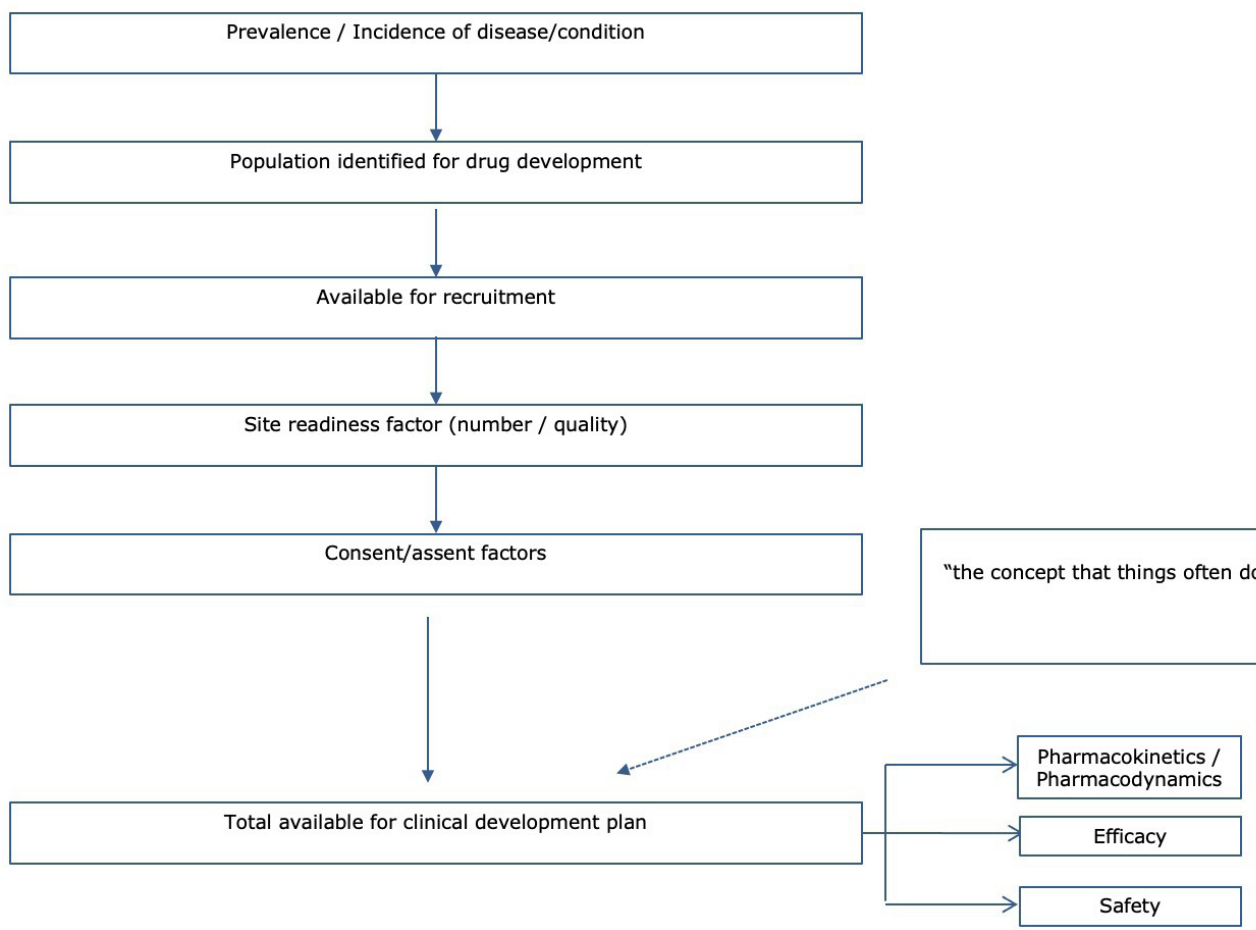
inform paediatric programmes and trials as appropriate. Cultivating relevant contacts in advance on the capabilities of paediatric clinical research networks means that questions can be posed rapidly. Standing arrangements with sites (confidential disclosure agreements) will facilitate timely work on preparedness. Clinical research networks can support preparedness by providing consistent relationships with a range of sites and rapid dissemination of requests for information and the collation of responses. Feedback to sites is valuable for them and to build relationships. When risks and hurdles are identified by sites during trial preparation, sponsors should not underestimate the risks and hurdles as they might reappear at a later stage of trial conduct, likely to be then a major constraint in the conduct of the trial.

Many clinical trials are or may become part of a regulatory drug development plan, such as a paediatric investigation plan (PIP) in the EU or UK, or a paediatric study plan (PSP) in the USA. When planning these studies, it is beneficial for sponsors to obtain regulatory input early on, and to keep an open dialogue on preparedness considerations. This can be done in the context of a PIP/PSP submission, and also other regulatory interactions (eg, scientific advice). ${ }^{25-28}$

\section{Improving the context for trial preparedness}

Other actions are needed beyond the preparation of individual trials.

In order to improve the landscape for medicines research, the paediatric community (clinicians, patients and families, regulators, ethicists, sponsors and CROs) needs to

1. Develop strategies to improve site selection.

2. Continue to undertake collaborative and constructive dialogue between patients' representatives, academics, industry and regulators to facilitate and accelerate treatment development for paediatric diseases, including rare diseases.

3. Tackle critical trial practicalities such as location of sites and travelling costs for participants and other ways of minimising the burden of research (such as virtual or home clinical trial visits and wearable technology).

4. Collect data that can be used to support and improve future trial preparation, including systematic collection of feedback from all involved (patients' representatives, researchers and academics, industry and regulators) to facilitate a culture of lessons learnt.

5. Lobby for greater recognition of the importance of research and readiness to participate in research among healthcare professionals and across society. Public and professional awareness around clinical trials needs to be improved, especially for paediatric trials.

6. Disseminate good practice across paediatric clinical research networks. Since the patients are often minors, there needs to be sensitivity to privacy, parental consent and data protection with such communication efforts.

7. Consider efficient, patient-focused trial designs and identify how global regulatory requirements have implications for preparation.

8. Promote transparency about results and preparation.

9. Develop understanding of the natural history and pathophysiology of conditions that can inform the definition of pharmaceutical targets.

There is a continued need for improved, mutual understanding of paediatric trial requirements and challenges across the regulatory network, companies, researchers and ethics committees as well as the public. This understanding is needed for efficient operations. More fundamentally, it is essential to respect the views and rights of participants in clinical trials. ${ }^{29}$ The intrinsic importance of this respect is supplemented by the need to retain trust among participants and the wider community. ${ }^{30}$ Working with children, young people and their parents is the best way to approach these issues. ${ }^{31}$ These issues that are not specific to individual products need a generic, precompetitive approach with contributions from multiple stakeholders. Research networks are well placed to support these broader issues, including Enpr-EMA. ${ }^{32} 33$

\section{CONCLUSIONS}

Lack of sufficient information during the preparation of trials often leads to their conduct being ineffective or to even fail. ${ }^{34}$ The effect of this inefficiency on the development of new drugs for the paediatric population has an important impact on the management of health of children since they often receive drugs which are not licensed or have even never been tested in their age group, or, even worse, have no available therapies for their disease. ${ }^{35-37}$ To help fill some gaps that contribute to the difficulty in implementing and conducting paediatric clinical trials, we have proposed an approach to collecting relevant information and a format for sharing that information. Everybody can contribute to preparedness in clinical trials, being a shared responsibility among the different players: some of the work to be prepared can be done upfront by the sites establishing their processes and procedures, by the sponsors opening communication channels with regulators, investigators and families, and by the patients organising in advocacy groups who can be representative of their voice also at regulatory level. Then, this work should be done as a collaborative effort exchanging knowledge and information and growing in experience. Preparedness does not apply only to the initial phase of the trial, but should be extended also to all the following phases, including the very last step on how to communicate the outcome of the study for regulatory purposes, for the scientific community and for the benefit of the participants. Explicit sharing of that information and assumptions when information is not available will promote rigorous preparation and facilitate the conduct of feasible and appropriate trials.

Collaborators The European Network of Paediatric Research at the European Medicines Agency Working Group on trial preparedness comprised: Angeliki Siapkara (lead), Cristina Seren, Tillmann Taube, Donato Bonifazi, Mark Turner, Ruth Ladenstein, Dimitrios Athanasiou, Solange Rohou, Pirkko Lepola, Gunter Egger, Claudio Fracasso, Siri Wang, Niyati Prasad, Segolene Galliard, Donato Bonifazi, Carmelo Rizzari, Loic Notelet, Niyati Prasad, Jackie O'Leary, Sabine Scherer and Margaret Patton.

Contributors AS led the Working Group and reviewed the manuscript. MAT drafted the manuscript. CF, GFE, CR, CST and DA reviewed the manuscript.

Funding The authors have not declared a specific grant for this research from any funding agency in the public, commercial or not-for-profit sectors.

Disclaimer The views expressed in this article are the personal views of the authors and may not be understood or quoted as being made on behalf of or reflecting the position of EMA or one of its committees or working parties.

Competing interests $\mathrm{CF}$ is an employee of Pfizer.

Patient consent for publication Not required.

Provenance and peer review Not commissioned; externally peer reviewed.

Data availability statement No data are available. Not applicable.

Open access This is an open access article distributed in accordance with the Creative Commons Attribution Non Commercial (CC BY-NC 4.0) license, which permits others to distribute, remix, adapt, build upon this work non-commercially, and license their derivative works on different terms, provided the original work is 
properly cited, appropriate credit is given, any changes made indicated, and the use is non-commercial. See: http://creativecommons.org/licenses/by-nc/4.0/.

\section{ORCID iD}

Mark A Turner http://orcid.org/0000-0002-5299-8656

\section{REFERENCES}

1 Lagler FB, Hirschfeld S, Kindblom JM. Challenges in clinical trials for children and young people. Arch Dis Child 2020.

2 Naka F, Strober B, Shahriari M. Clinical trials: kids are not just little people. Clin Dermatol 2017:35:583-93.

3 EC. State of paediatric medicines in the EU: 10 years of the EU paediatric regulation. Available: https://ec.europa.eu/health/sites/health/files/files/paediatrics/docs/2017_ childrensmedicines_report_en.pdf [Accessed 14 Mar 2021].

4 Turner MA, Catapano M, Hirschfeld S, et al. Paediatric drug development: the impact of evolving regulations. Adv Drug Deliv Rev 2014;73:2-13.

5 Mentzer D. Progress review of the European paediatric regulatory framework after six years of implementation. Int J Pharm 2014;469:240-3.

6 Tsukamoto K, Carroll KA, Onishi T, et al. Improvement of pediatric drug development: regulatory and practical frameworks. Clin Ther 2016;38:574-81.

7 Toma M, Felisi M, Bonifazi D, et al. Paediatric medicines in Europe: the paediatric Regulation-Is it time for reform? Front Med 2021;8:593281.

8 Bucci-Rechtweg C. Enhancing the pediatric drug development framework to deliver better pediatric therapies tomorrow. Clin Ther 2017;39:1920-32.

9 Caldwell PHY, Murphy SB, Butow PN, et al. Clinical trials in children. Lancet 2004;364:803-11.

10 Group EE. Ethical considerations for clinical trials on medicinal products conducted with minors. recommendations of the expert group on clinical trials for the implementation of regulation (EU) NO 536/2014 on clinical trials on medicinal products for human use. Available: https://ec.europa.eu/health/sites/health/files/files/ eudralex/vol-10/2017_09_18_ethical_consid_ct_with_minors.pdf [Accessed $14 \mathrm{Mar}$ 2021].

11 McCullough MB, Janicke D, Odar Stough C, et al. Barriers to recruitment in pediatric obesity trials: comparing Opt-in and Opt-out recruitment approaches. J Pediatr Psychol 2017:42:174-85.

12 Greenberg RG, Gamel B, Bloom D, et al. Parents' perceived obstacles to pediatric clinical trial participation: findings from the clinical trials transformation initiative. Contemp Clin Trials Commun 2018;9:33-9.

13 Hoberman A, Shaikh N, Bhatnagar S, et al. Factors that influence parental decisions to participate in clinical research: consenters vs nonconsenters. JAMA Pediatr 2013;167:561-6.

14 Institute of Medicine Committee on Clinical Research Involving C. The National Academies Collection: Reports funded by National Institutes of Health. In: Field MJ, Behrman RE, eds. Ethical conduct of clinical research involving children. Washington (DC): National Academies Press (US) Copyright (C) 2004, National Academy of Sciences, 2004.

15 Wang S, Huemer KH. Paediatric pharmaceutical legislation and its impact on adult and paediatric drug development: The EU regulatory view. In: Bar-Shalom D, Rose K, eds. Pediatric formulations: a roadmap. Springer Verlag, 2014: 395-404.

16 Saint Raymond A. Regulatory aspects of drug development in children: change and resistance to change. Expert Rev Clin Pharmacol 2010;3:593-5.

17 Auby P. Pharmaceutical research in paediatric populations and the new EU paediatric legislation: an industry perspective. Child Adolesc Psychiatry Ment Health 2008;2:38.

18 England A, Wade K, Smith PB, et al. Optimizing operational efficiencies in early phase trials: the pediatric trials network experience. Contemp Clin Trials 2016;47:376-82.
19 Turner MA. Clinical trials of medicines in neonates: the influence of ethical and practical issues on design and conduct. Br J Clin Pharmacol 2015;79:370-8.

20 European th medicines Agency (Enpr-EMA). European network of paediatric research. Available: https://www.ema.europa.eu/en/partners-networks/networks/europeannetwork-paediatric-research-european-medicines-agency-enpr-ema [Accessed 15 Dec 2020].

21 EMA. Preparedness of medicines' clinical trials in paediatrics: Recommendations by the Enpr-EMA working group on trial preparedness. Available: https://www.ema. europa.eu/en/documents/other/preparedness-medicines-clinical-trials-paediatricsrecommendations-enpr-ema-working-group-trial_en.pdf [Accessed 18 Mar 2021].

22 EMA. Mandate of Enpr-EMA working groups. Available: https://www.ema.europa. eu/en/documents/other/mandate-european-network-paediatric-research-europeanmedicines-agency-working-groups_en.pdf [Accessed 15 Dec 2020].

23 Williams K, Thomson D, Seto I, et al. Standard 6: age groups for pediatric trials. Pediatrics 2012;129:\$153-60.

24 Van't Hoff W, Offringa M, Star Child Health group. Star child health: developing evidence-based guidance for the design, conduct and reporting of paediatric trials. Arch Dis Child 2015;100:189-92.

25 EMA. Paediatric investigation plans: templates, forms and submission dates. Available: https://www.ema.europa.eu/en/human-regulatory/research-development/paediatricmedicines/paediatric-investigation-plans/paediatric-investigation-plans-templatesforms-submission-dates [Accessed 18 Mar 2021].

26 EMA. Scientific advice and protocol assistance. Available: https://www.ema.europa. eu/en/human-regulatory/research-development/scientific-advice-protocol-assistance [Accessed 18 Mar 2021].

27 EMA. Prime: priority medicines. Available: https://www.ema.europa.eu/en/humanregulatory/research-development/prime-priority-medicines

28 FDA. Pediatric study plans: content of and process for Submitting initial pediatric study plans and amended initial pediatric study plans. Available: https://www.fda. gov/regulatory-information/search-fda-guidance-documents/pediatric-study-planscontent-and-process-submitting-initial-pediatric-study-plans-and-amended [Accessed 18 Mar 2021].

29 Dorscheidt JHHM, Hein IM. Medical Research Involving Children - Giving Weight to Children's Views. Int J Child Rights 2018;26:93-116.

30 Bioethics NCf. Children and clinical research: ethical issues. Available: https://www. nuffieldbioethics.org/assets/pdfs/Children-and-clinical-research.pdf [Accessed $18 \mathrm{Mar}$ 2021].

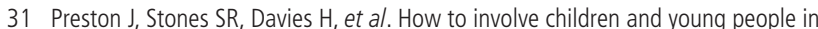
what is, after all, their research. Arch Dis Child 2019;104:494-500.

32 Turner MA, Hildebrand H, Fernandes RM, et al. The conect4children (C4c) Consortium: potential for improving European clinical research into medicines for children. Pharmaceut Med 2021;35:71-9.

33 EMA. European network of paediatric research at the European medicines Agency (Enpr-EMA). Available: https://www.ema.europa.eu/en/partners-networks/networks/ european-network-paediatric-research-european-medicines-agency-enpr-ema [Accessed 18 Mar 2021].

34 Hwang TJ, Tomasi PA, Bourgeois FT. Delays in completion and results reporting of clinical trials under the paediatric regulation in the European Union: a cohort study. PLoS Med 2018;15:e1002520.

35 The Lancet . Prescribing off-label drugs for children: when will it change? Lancet 2019:394:1114

36 Hoon D, Taylor MT, Kapadia P, et al. Trends in off-label drug use in ambulatory settings: 2006-2015. Pediatrics 2019;144:144

37 Bazzano ATF, Mangione-Smith R, Schonlau M, et al. Off-Label prescribing to children in the United States outpatient setting. Acad Pediatr 2009;9:81-8. 